\title{
Mutant alleles of FAD2-1A and FAD2-1B combine to produce soybeans with the high oleic acid seed oil trait
}

\author{
Anh-Tung Pham', Jeong-Dong Lee ${ }^{2}$, J Grover Shannon ${ }^{3}$, Kristin D Bilyeu ${ }^{4 *}$
}

\begin{abstract}
Background: The alteration of fatty acid profiles in soybean [Glycine max (L.) Merr.] to improve soybean oil quality is an important and evolving theme in soybean research to meet nutritional needs and industrial criteria in the modern market. Soybean oil with elevated oleic acid is desirable because this monounsaturated fatty acid improves the nutrition and oxidative stability of the oil. Commodity soybean oil typically contains $20 \%$ oleic acid and the target for high oleic acid soybean oil is approximately $80 \%$ of the oil; previous conventional plant breeding research to raise the oleic acid level to just $50-60 \%$ of the oil was hindered by the genetic complexity and environmental instability of the trait. The objective of this work was to create the high oleic acid trait in soybeans by identifying and combining mutations in two delta-twelve fatty acid desaturase genes, FAD2-1A and FAD2-1B.

Results: Three polymorphisms found in the FAD2-1B alleles of two soybean lines resulted in missense mutations. For each of the two soybean lines, there was one unique amino acid change within a highly conserved region of the protein. The mutant FAD2-1B alleles were associated with an increase in oleic acid levels, although the FAD2-1B mutant alleles alone were not capable of producing a high oleic acid phenotype. When existing FAD2-1A mutations were combined with the novel mutant FAD2-1B alleles, a high oleic acid phenotype was recovered only for those lines which were homozygous for both of the mutant alleles.

Conclusions: We were able to produce conventional soybean lines with $80 \%$ oleic acid in the oil in two different ways, each requiring the contribution of only two genes. The high oleic acid soybean germplasm developed contained a desirable fatty acid profile, and it was stable in two production environments. The presumed causative sequence polymorphisms in the FAD2-1B alleles were developed into highly efficient molecular markers for tracking the mutant alleles. The resources described here for the creation of high oleic acid soybeans provide a framework to efficiently develop soybean varieties to meet changing market demands.
\end{abstract}

\section{Background}

Soybean oil consumed in the U.S. accounted for approximately $70 \%$ of the total edible fat and oil consumption in 2008; three quarters of the soybean oil was used as cooking oil and baking and frying fat (http:// www.soystats.com/2009/). Soybean oil's utilization is determined by its fatty acid composition, with commodity soybean oil typically containing $13 \%$ palmitic acid (16:0), 4\% stearic acid (18:0), 20\% oleic acid (18:1), 55\%

\footnotetext{
* Correspondence: Kristin.Bilyeu@ars.usda.gov

${ }^{4}$ USDA-ARS, Plant Genetics Research Unit, 110 Waters Hall, Columbia, MO 65211 USA

Full list of author information is available at the end of the article
}

linoleic acid (18:2), and 8\% linolenic acid (18:3). Consumption of oils with high oleic acid content is desirable because this monounsaturated fatty acid not only improves shelf life but also reduces the need for hydrogenation, a process adding to the cost of the oil and generating unwanted trans-fat that has been linked to many health problems in humans [1]. Additionally, in biodiesel production, there is also a need for oils high in oleic acid and low in saturated fatty acids in order to improve the oxidative stability while augmenting cold flow [2]. Moreover, the enhanced oxidative stability of soybean oil with higher oleic acid content will also open
C Biomed Central

C 2010 Pham et al; licensee BioMed Central Ltd. This is an Open Access article distributed under the terms of the Creative Commons Attribution License (http://creativecommons.org/licenses/by/2.0), which permits unrestricted use, distribution, and reproduction in any medium, provided the original work is properly cited. 
up a variety of food uses and industrial applications like spraying oils or machine lubricants [3].

In the lipid biosynthetic pathway, conversion of oleic acid (18:1) precursors to linoleic acid (18:2) precursors is catalysed by the delta-twelve fatty acid desaturase 2 enzyme (FAD2) [4,5]. While in Arabidopsis and maize only one single copy of a FAD2 gene was identified $[4,6]$, soybean appears to possess multiple copies of the gene due to the consequence of repeated rounds of genome duplication followed by limited sequence loss [5]. The soybean FAD2 gene family has been previously characterized at the genome level for structure and expression [5]. Among the FAD2 genes identified in soybean, the FAD2-2 desaturases consisting of FAD2-2A (Glyma19g32930), FAD2-2B (Glyma19g32940), and FAD2-2C (Glyma03g30070) were found to be widely expressed in the vegetative tissues of the soybean plant [5]. The exception was $F A D 2-2 A$, for which expression was not detected; $F A D 2-2 A$ was predicted to be nonfunctional as it has a deletion of $100 \mathrm{bp}$ in the coding region [5]. The two microsomal FAD2-1 desaturases FAD2-1A (Glyma10g42470) and FAD2-1B (Glyma20g24530) were mainly expressed in developing seeds $[5,7]$. Thus, $F A D 2-1 A$ and $F A D 2-1 B$ are considered to play an important role in controlling the oleic acid level in developing soybean seeds and were selected as candidate genes to elucidate the molecular genetic basis of soybean lines from the germplasm collection that contained elevated levels of oleic acid.

$F A D 2-1 A$ and $F A D 2-1 B$ are most closely related to one another, with a shared genomic organization containing a single intron and 99\% identity in encoded amino acid sequence, and are present on homologous chromosome regions mapped to linkage group $\mathrm{O}$ (chromosome 10) and I (chromosome 20), respectively [5,7]. Characterization of the expression of the individual soybean FAD2 genes confirmed the importance of FAD2$1 A$ and $F A D 2-1 B$ with expression of these genes during peak oil synthesis; a possible role was also revealed for FAD2-2C under cool temperature conditions [5]. Differential response to temperature was also demonstrated for the soybean FAD2-1A and FAD2-1B enzymes expressed in yeast [7]. The temperature during soybean pod fill has been shown to influence the fatty acid composition of soybean oil, with cooler temperatures leading to increased oleic acid desaturation and decreased oleic acid accumulation [8-10].

Selection for breeding and genetic engineering resulting in elevated oleic acid levels were reported in many oilseed crops: safflower[11], sunflower [12], peanut [13-15], canola $[16,17]$, cotton [12] and maize [6]. While the elevated oleic acid phenotype was often observed after a single $F A D 2$ gene was mutated, the very highest oleic acid phenotypes ( $>80 \%$ of the total oil) were achieved most frequently by silencing all copies of FAD2 genes that were expressed in developing seeds, or in particular for peanut, by combining the mutation in the active site of ahFAD2A with the loss of transcription of $a h F A D 2 B[14,15]$. No soybean lines exist in the USDA National Plant Germplasm System collection with the high oleic acid trait (oleic acid content above $70 \%$ of the oil fraction), although multiple lines contain elevated oleic acid levels [9].

Several elevated oleic acid soybean lines have been characterized at the molecular level. The destruction of the FAD2-1A gene by $\mathrm{X}$-ray mutagenesis yielded two soybean lines with oleic acid content of approximately $50 \%$ of the oil [18-20]. A reverse genetics approach was utilized to identify a soybean line containing a missense mutation in FAD2-1A that associated with an elevated oleic acid content of the oil [21]. Many soybean lines were developed through recurrent selection that contained elevated oleic acid content such as N00-3350, N98-4445A, and N97-3363-3 [22], but the genetic basis for the trait was extremely complex, with at least six QTLs conditioning the phenotype [23,24]. In addition, the level of oleic acid in the oil of these soybean lines was particularly susceptible to environmental effects when compared to the X-ray FAD2-1A deletion line $[9,10]$. No mutations in $F A D 2-1 B$ that associate with elevated oleic acid content have been reported to date.

Suppression of FAD2-1 gene expression by means of genetic engineering has been successful in creating soybean lines with the high oleic acid trait, with oleic acid content above $80 \%$ of the oil fraction reported and very little environmental impact on the trait $[3,25,26]$. Transgenic expression of ribozyme terminated sense or antisense $F A D 2-1$ constructs was successful in eliminating the FAD2-1 mRNA expression signal in developing embryos and producing siRNAs for the FAD2-1 genes [25]. No attempt was made to distinguish between the $F A D 2-1 A$ and $F A D 2-1 B$ genes in the transgenic work, and it is also possible that the FAD2-2 genes were targeted in the experiments [25].

The objective of this work was to create the high oleic acid trait in soybeans using conventional plant breeding technology. We hypothesized that combinations of mutant alleles of the soybean FAD2-1A and FAD2-1B genes would greatly reduce the FAD2 enzyme activity in developing seeds and thus result in an accumulation of oleic acid at the expense of linoleic and linolenic acid in the triacylglycerol fraction of the seed oil. We took a candidate gene approach with the FAD2-1A and FAD2$1 B$ genes present in soybean germplasm accessions containing elevated levels of oleic acid in the oil. Two mutant alleles of $F A D 2-1 B$ were identified that associated with elevated oleic acid content. In addition, mutant alleles of $F A D 2-1 A$ and $F A D 2-1 B$ were combined to create soybeans with the high oleic acid trait. 


\section{Results \\ Identification of mutant alleles of $F A D 2-1 B$ in soybean lines PI 283327 and PI 567189A}

In an effort to identify novel alleles of the soybean $F A D 2-$ $1 A$ and $F A D 2-1 B$ genes, genomic DNA was characterized for the sequence of both genes from plant introduction (PI) lines selected from the National Genetic Resources Program containing elevated oleic acid levels from 27 to approximately $50 \%$ percent of the oil, while commodity soybeans typically produce $19-23 \%$ oleic acid [27]. The FAD2-1A alleles from PI 283327 and PI 567189 A were identical to the reference 'Williams 82 ' [28] allele. In contrast, for the FAD2-1B genes from PI 283327 and PI 567189 A, seven common single nucelotide polymorphisms (SNPs) and one unique SNP for each line were identified when compared to the reference sequences from the cultivar 'Williams 82' (Figure 1A). Other soybean lines were also characterized for their FAD2-1B alleles, and two independent lines, PI 210179 and PI 578451, contained exactly the same FAD2-1B alleles as PI 283327 and PI 567189 A, respectively.

For the PI 283327 FAD2-1B allele, there were three missense mutations resulting in S86F, M126V, and P137R (Figure 1B). For the PI 567189 A FAD2-1B allele, two of the missense mutations were identical (S86F and M126V), and the third missense mutation was unique, I143T. Other soybean lines were identified that had $F A D 2-1 B$ alleles which contained different combinations of the silent mutations and the S86F and M126V missense mutations (data not shown).

To predict the potential effect of the amino acid changes to soybean FAD2-1B enzyme function, we used the program PolyPhen to analyze the potential severity of each amino acid change [29]. In addition, the relative amino acid conservation for each position in the enzyme was evaluated visually using Weblogo after alignment of 100 different FAD2 protein sequences present in the National Center for Biotechnology Information database [30]. The two shared amino acid substitutions M126V and S86F each occur at a highly variable position according to the alignment in Weblogo and were classified by Polyphen as benign substitutions, indicating they are likely to have no phenotypic effect. For the M126V change, methionine and valine have similar chemical properties.

$F A D 2-1 B$ alleles that encoded only the S86F misssense mutation were identified and analyzed for the functional consequence of that isolated mutation. Although serine and phenylalanine are amino acids with different chemical properties, several lines of evidence indicated that the S86F FAD2-1B allele was functional, including detection of delta-twelve fatty acid desaturase activity in a yeast recombinant expression experiment and no association of the S86F FAD2-1B allele variant with an elevated oleic acid phenotype (data not shown).

The FAD2-1B P137R mutation present in PI 283327 represents a charge change for the substituted amino acid since proline is nonpolar while arginine is basic. At position 137 of wild-type FAD2-1B, the proline is perfectly conserved; a proline at that position is invariant for all of the tested FAD2 sequences represented in the protein database (Figure 1C). In PolyPhen, the amino acid change was classified as probably damaging for P137R, which means that this SNP is predicted to affect protein structure and/or function.

For the isoleucine present at position 143 of wild-type FAD2-1B, other amino acids were observed in this position in the FAD2 sequences present in the protein database, indicating that an isoleucine at position 143 is less conserved than the proline at the 137 position (Figure 1C). However, the substitution of threonine for isoleucine at this position was not observed in the database analysis. Moreover, isoleucine and threonine also have contrasting chemical properties since isoleucine is nonpolar while threonine is uncharged polar. In PolyPhen, the I143T amino acid change was classified as probably damaging. The conservation of amino acids in the general region of the $F A D 2-1 B$ P137R and I143T mutations combined with the chemical nature of the changes is suggestive of the potential deleterious effects of these mutations to the FAD2-1B enzyme's structure and function.

\section{The PI 283327 FAD2-1B allele is associated with an increase in seed oleic acid content}

To test the hypothesis that the newly identified mutations in $F A D 2-1 B$ are causative for the elevated oleic acid level in the plant introduction lines, an analysis of the oleic acid phenotype and $F A D 2-1 B$ genotype association was examined for Population 1 , an $\mathrm{F}_{6}$ recombinant inbred line (RIL) population developed from the cross 'Jake'[31] × PI 283327. The commodity soybean line Jake typically produces approximately $22 \%$ oleic acid in the seed oil and contains functional FAD2-1A and $F A D 2-1 B$ alleles, represented as genotype FAD2-1 AABB. PI 283327 was selected from the germplasm collection because of elevated levels of oleic acid in the seed oil and carries the mutant FAD2-1B P137R allele, represented as genotype $F A D 2-1$ AAbb with the lowercase allele designation always specifying the mutant allele and the capital case specifying the wild-type allele. Of the 54 lines in the RIL population 1 that contained homozygous alleles of $F A D 2-1 B$, the 30 lines carrying the mutant FAD2-1B P137R alleles from PI 283327 had an average of $29.4 \%$ oleic acid, while 24 lines carrying wild type alleles had an average of $20.5 \%$ oleic acid 
A.

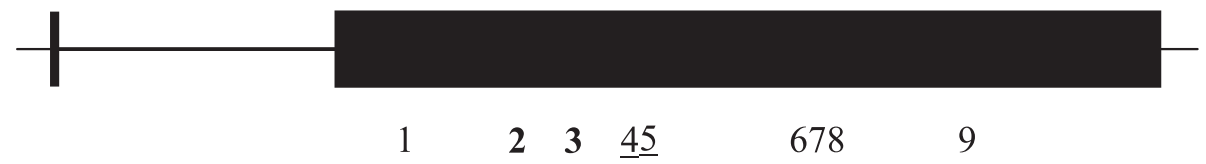

B.

\begin{tabular}{|c|c|c|}
\hline PI 283327 & 61 & VVYDLSLAFIFYIATTYFHLLPHPFFLIAWP IYWVLQGCILTGVWVIAHECGHHAFSKYP \\
\hline PI $567189 \mathrm{~A}$ & 61 & VVYDLSLAFIFYIATTYFHLLPHPFFLIAWP IYWVLQGCILTGVWVIAHECGHHAFSKYP \\
\hline Williams 82 & 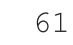 & PESLIAWP IYWVLQGCILTGVWVIAHECGHHAFSKY \\
\hline 83327 & 12 & TVHSALLVRYFSWKISHRRHHSNTGSLDRDEVFVPKPKSKVAWYTKYLNNPI \\
\hline $7189 \mathrm{~A}$ & 121 & WVDDV GLTVHSALLVPYFSWKTSHRRHHSNTGSLDRDEVFVPKPKSKVAWYTKYLNNP \\
\hline illiams 82 & 121 & WVDDVMGLTVHSALLVPYFSWKISHRRHHSNTGSLDRDEVFVPKPKSKVAWYTKYLNNP \\
\hline
\end{tabular}

C.

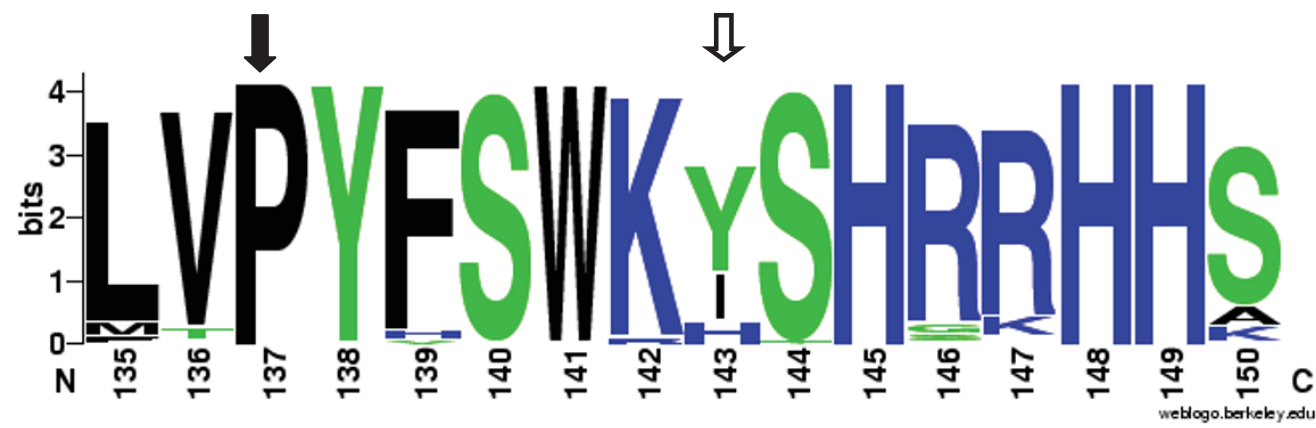

Figure 1 Characterization of mutations in the FAD2-1B alleles from soybean lines PI 283327 and PI 567189A. A. Schematic of the FAD2IB gene and the polymorphisms identified in the alleles from PI 283327 and PI 567189A. The horizontal line represents the DNA for FAD2-1B, with the heavier line indicating the intron and lighter lines representing the $5^{\prime}$ and $3^{\prime}$ untranslated portions of the gene. The dark vertical line represents the portion of exon 1 that contains only the start codon and the darkened rectangle represents exon 2. Numbers beneath the schematic indicate the positions of polymorphisms compared to the Williams 82 reference FAD2-1B coding sequence, with shared missense mutations in bold and non-shared missense mutations underlined. $1=\mathrm{a} 105 \mathrm{~g}$, silent; $2=\mathrm{c} 257 \mathrm{t}, \mathrm{S} 86 \mathrm{~F} ; 3=\mathrm{a} 376 \mathrm{~g}, \mathrm{M} 126 \mathrm{~V} ; 4=\mathrm{c} 410 \mathrm{~g}, \mathrm{P} 137 \mathrm{R}$ unique to PI 283327 and PI 210179; 5 = t428c, 1143T unique to PI 567189A and PI 578451; 6 = c657t, silent; $7=$ t669c, silent; $8=$ t682c, silent; 9 $=a 918 \mathrm{~g}$, silent. $\mathbf{B}$. Fatty acid desaturase FAD2-1B amino acid sequence alignment in the region surrounding the mutations in PI 283327 and $\mathrm{PI}$ 567189 A. Amino acid positions are indicated at the beginning of each line of the alignment. Identical amino acid residues are highlighted in black, a similar amino acid substitution is highlighted in gray, and the S86F, P137R, and I143T mutations are not highlighted. C. Weblogo output of the amino acid conservation FAD2 enzyme as part of the BLINK feature at NCBI using GI number 197111722 . The top 100 best matched sequences were aligned and used as input for sequence LOGO http://weblogo.berkeley.edu/logo.cgi. The logo consists of stacks of symbols, one stack for each position in the amino acid sequence. The overall height of the stack indicates the sequence conservation at that position, while the height of symbols within the stack indicates the relative frequency of each amino acid at that position. Closed arrow indicates residue changed due to the P137R FAD2-1B mutation in PI 283327 and open arrow indicates residue changed due to the I143T FAD2-1B mutation in PI 567189A. 
(Figure 2). Although the variation in the data was large, the difference in oleic acid contents between the two contrasting $F A D 2-1 B$ genotypes was confirmed significant using Student's t-test at the 0.05 probability level $(\mathrm{P}>0.05)$

\section{Combinations of mutations in FAD2-1A and FAD2-1B produce high oleic acid levels in the seed oil}

We hypothesized that combining the mutant alleles of both $F A D 2-1 A$ and $F A D 2-1 B$ in one soybean line would eliminate most of the enzyme activity responsible for converting oleic acid precursors into linoleic acid precursors, and thus result in a higher oleic acid content compared to soybean lines containing mutations in either gene individually. Two mutations in FAD2-1A were available: the deletion of the $F A D 2-1 A$ gene in soybean line M23 (designated herein as FAD2-1A $\Delta$ ) [18] and the missense mutation in FAD2-1A from line 17D (designated herein as FAD2-1A S117N) [21]. The identification of the two missense mutant alleles in $F A D 2-1 B$ from PI 283327 (FAD2-1B P137R) and PI 567189A (FAD2-1B I143T) created the opportunity to evaluate the oleic acid phenotype in soybean lines containing different combinations of mutant FAD2-1A and FAD2-1B alleles.

An association analysis of the oleic acid phenotype and the $F A D 2-1 A$ and $F A D 2-1 B$ genotypes was performed for Population 2, a RIL population consisting of $\mathrm{F}_{2: 6}$ and $\mathrm{F}_{2: 7}$ lines developed from the cross $\mathrm{M} 23 \times \mathrm{PI}$ 283327 and grown in Portageville MO in 2008. Since

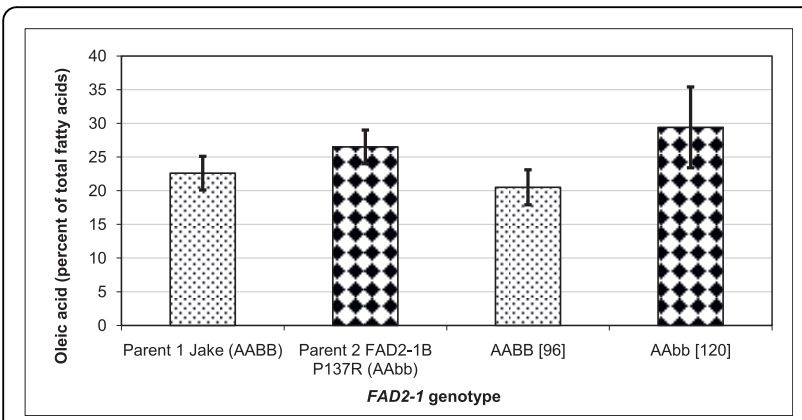

Figure 2 Seed oleic acid phenotype and FAD2-1 genotype association analysis for population 1 . Average oleic acid content of soybean seeds produced in Portageville, MO from the $\mathrm{F}_{6}$ RILs developed from the cross Jake $\times$ PI 283327, population 1. Labels on the $X$-axis include: two parents with contrasting FAD2-1B genotypes and the RILs grouped by FAD2-1B genotype. BB indictates wild-type FAD2-1B alleles, and bb indicates mutant P137R FAD2-1B alleles derived from PI 283327; brackets surround the number of soybean seed samples represented in the genotype class. Error bars indicate plus and minus one standard deviation from the mean. Oleic acid phenotype data of each genotype class is the mean of oleic acid content as a percentage of the total fatty acid content of the oil of all lines carrying the FAD2-1 genotype, four individual samples for each line. soybean line M23 contained FAD2-1A $\Delta$ and wild-type alleles of $F A D 2-1 B$, the genotype is herein represented as $F A D 2-1$ aaBB with the lowercase allele designation always specifying the mutant allele and the capital case specifying the wild-type allele; likewise, the FAD2-1 P137R genotype of PI 283327 is represented here as FAD2-1 AAbb. Individual seeds from each of 40 lines produced in an appropriate field environment and carrying the different homozygous combinations of FAD2-1A and $F A D 2-1 B$ were analyzed along with the parental lines for the fatty acid phenotype of the seed oil (Figure 3). Transgressive segregation for oleic acid content was observed for the lines that inherited the FAD2-1 AABB and aabb genotypes, while the lines that recovered the parental $F A D 2-1$ genotypes contained oleic acid contents similar to the phenotype of the parental lines. Lines with the genotype FAD2-1 AABB had an average oleic acid content similar to that of a conventional soybean 'Jake', which was $22.6 \%$ of total oil

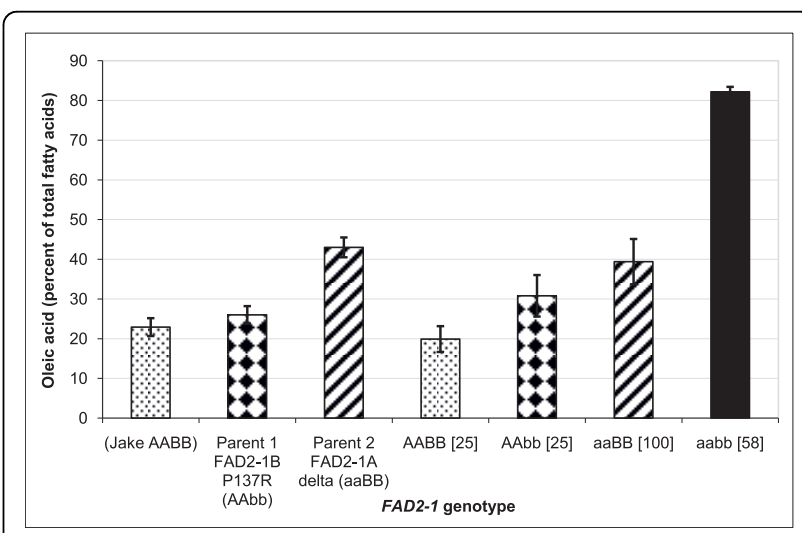

Figure 3 Seed oleic acid phenotype and FAD2-1 genotype association analysis for population $\mathbf{2}$. Average oleic acid content of soybean seeds produced in Portageville, $\mathrm{MO}$ from the segregating $F_{6}$ and $F_{7}$ RILs developed from the cross $\mathrm{M} 23 \times \mathrm{PI}$ 283327, population 2. X-axis labels indicate a typical commodity soybean line (Jake), the two RIL parents, and the RILs grouped by $F A D 2-1 A$ and $F A D 2-1 B$ genotype. $A A=$ wild-type $F A D 2-1 A$ alleles, aa = mutant FAD2-1A $\triangle$ alleles derived from M23, BB = wild-type FAD2$1 B$ alleles, $b b=$ mutant P137R FAD2-1B alleles derived from PI 283327; brackets surround the number of soybean seed samples represented in the genotype class. Error bars indicate plus and minus one standard deviation from the mean. Oleic acid phenotype data of each genotype class is the mean of oleic acid content as a percentage of the total fatty acid content of the oil of all lines carrying the FAD2-1 genotype, five individual samples for each line, with two exceptions, one for class aaBB, where six lines had ten seed samples and eight lines had five seed samples. Additionally, two separate individual seed samples from the aabb lines were ommitted from the analysis because their genotypes could not be verified. Including these two samples would have led to a mean of $80.8 \pm 8.0 \%$ oleic acid for the FAD2-1 aabb genotype class.

Subsequent sampling of ten additional seeds from these lines produced oleic acid levels with a range of $81.5 \%$ to $86.2 \%$ oleic acid in the seed oil. 
content. In contrast, individuals with the genotype FAD2-1 aabb had an average of $82.2 \%$ oleic acid, with a very narrow standard deviation of $1.2 \%$. In this population, lines with either homozygous mutant FAD2-1A or $F A D 2-1 B$ alleles had an average of $39.4 \%$ and $30.6 \%$ oleic acid, respectively, reiterating the relatively minor increase in oleic acid level conditioned by the $F A D 2-1 B$ alleles in the presence of functional FAD2-1A alleles.

A similar experiment investigated the impact on seed oleic acid levels for the genetic combination of the $F A D 2-1 A \triangle$ alleles from M23 with the alternate FAD2$1 B$ I143T alleles present in PI 567189 A. For Population 3, a M23 × PI 567189A RIL population, we genotyped lines based on the fatty acid profiles of $F_{5}$ seeds harvested in Costa Rica, and then selected to present here the 31 lines classified into four homozygous combinations of $F A D 2-1 A$ and $F A D 2-1 B$. The average oleic acid contents of the four $F A D 2-1$ genotypes were significantly different from each other and again demonstrated transgressive segregation (Figure 4). Lines with FAD2-1 genotype $\mathrm{AABB}$ had an average oleic acid content not statistically different from that of Jake. In contrast, soybean lines with the FAD2-1aabb genotype had an average of $80.3 \%$ oleic acid. The lines that recovered the parental FAD2-1 genotypes also recovered the respective parental oleic acid phenotype.

Soybean line $17 \mathrm{D}$ was discovered in a reverse genetics screen for mutations in FAD2-1A [21]. Line 17D contains elevated oleic acid in the seed oil due to a FAD2$1 \mathrm{~A}$ missense mutation in a conserved amino acid referred to here as FAD2-1A S117N. Soybean lines which contained the $F A D 2-1 A$ S117N alleles consistently accumulated lower oleic acid levels in the seed oil than lines containing FAD2-1A $\Delta$ alleles derived from M23, and the phenotype was not stable in different environments [21]. We next examined the combining ability in Population 4, the $\mathrm{F}_{2}$ and $\mathrm{F}_{2: 3}$ individuals from the cross of the $F A D 2-1 A \mathrm{~S} 117 \mathrm{~N}$ alleles derived from line 17D with the FAD2-1B P137R alleles derived from PI 283327 $(17 \mathrm{D} \times \mathrm{S} 08-14788)$. Homozygous FAD2-1A and FAD2$1 B$ allele combinations were selected from $F A D 2-1$ genotyped $\mathrm{F}_{2}$ plants for field growth in an appropriate environment and subsequent $\mathrm{F}_{3}$ seed oil fatty acid phenotype determination (Figure 5). Transgressive segregation was observed for the genotypes that inherited both the homozygous wild-type FAD2-1 alleles and the homozygous mutant FAD2-1 allele combinations. The FAD2-1 aabb combination demonstrated an average

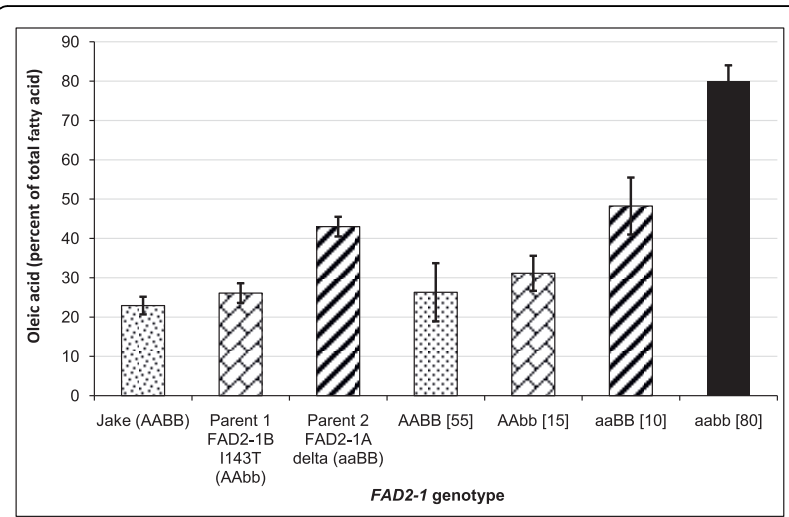

Figure 4 Seed oleic acid phenotype and FAD2-1 genotype association analysis for population 3. Average oleic acid content of soybean seeds produced in Costa Rica from the segregating $\mathrm{F}_{6}$ and $F_{7}$ RIL developed from the cross M23 $\times$ PI 567189 A population 3. $X$-axis labels indicate a typical commodity soybean line (Jake), the two RIL parents, and the RILs grouped by FAD2-1A and FAD2-1B genotype. $A A=$ wild-type $F A D 2-1 A$ alleles, aa = mutant FAD2-1A $\triangle$ alleles derived from $M 23, B B=$ wild-type $F A D 2-1 B$ alleles, $b b=$ mutant I143T FAD2-1B alleles derived from PI 567189 A; brackets surround the number of soybean seed samples represented in the genotype class. Error bars indicate plus and minus one standard deviation from the mean. Oleic acid phenotype data of each genotype class is the mean of oleic acid content as a percentage of the total fatty acid content of the oil of all lines carrying the FAD2-1 genotype, five individual samples for each line, with the exception of the aaBB class, for which there was only one line and ten individual seeds were analyzed.

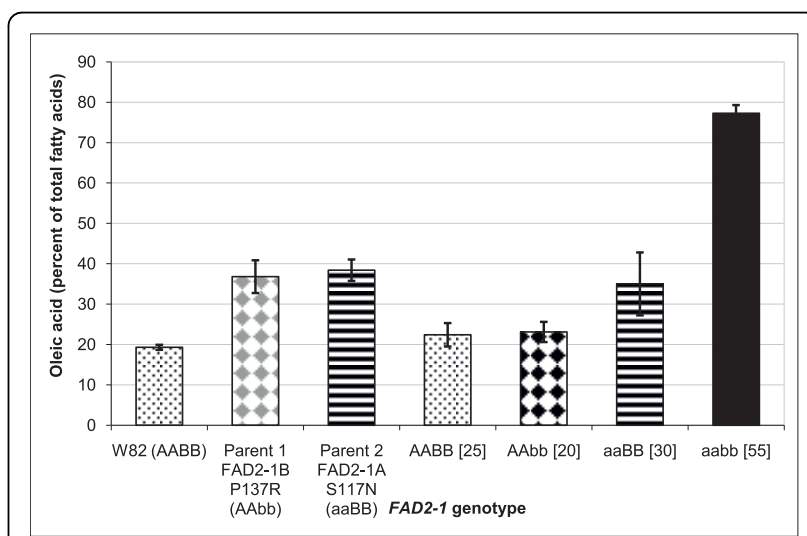

Figure 5 Seed oleic acid phenotype and FAD2-1 genotype association analysis for $F_{3}$ seeds of population 4 . Average oleic acid content of soybean seeds produced in Columbia, $\mathrm{MO}$ from the segregating $F_{2}$ population developed from the cross $17 \mathrm{D} \times \mathrm{S} 08$ 14788, population 4. X-axis labels indicate a typical commodity soybean line (Williams 82, W82), the two RIL parents, and the RILs grouped by FAD2-1A and FAD2-1B genotype. $A A=$ wild-type $F A D 2$ $1 A$ alleles, aa $=$ mutant S117N FAD2-1A alleles derived from 17D, BB = wild-type $F A D 2-1 B$ alleles, $b b=$ mutant P137R FAD2-1B alleles derived from PI 283327; brackets surround the number of soybean seed samples represented in the genotype class. Error bars indicate plus and minus one standard deviation from the mean. Oleic acid phenotype data of each genotype class is the mean of oleic acid content as a percentage of the total fatty acid content of the oil of all lines carrying the FAD2-1 genotype, five individual samples for each line. 
oleic acid content of 77.3\%; the AABB combination displayed a typical commodity soybean oleic acid level. The parental oleic acid phenotype was recovered for the FAD2-1 aaBB genotype but not for the FAD2-1 AAbb genotype.

\section{Excess desaturase activity: A single wild-type FAD2-1 allele prevents high oleic acid accumulation}

Our initial investigation of both the FAD2-1 genotype and fatty acid phenotype in $\mathrm{F}_{2}$ seeds from Population 4 (FAD2-1A S117N × FAD2-1B P137 cross) demonstrated the epistatic nature of the mutant alleles working in combination, and the results revealed that only homozygous combinations of both mutant FAD2-1A and FAD2$1 B$ were capable of producing the high oleic acid phenotype. Of the $200 \mathrm{~F}_{2}$ seeds that were phenotyped, there were 12 individual $\mathrm{F}_{2}$ seeds with genotype FAD2-1 aabb, and they had an average oleic acid content of $81 \%$, ranging from $75.2 \%$ to $83.9 \%$ oleic acid (Figure 6 ). The next highest oleic acid phenotype in the set was $48.8 \%$, and that seed had the FAD2-1 Aabb genotype. For a two recessive gene model, one sixteenth of the individuals should inherit the phenotype; recovery of 12 individuals with the high oleic acid phenotype satisfies this expectation by Chi-Square test at the 0.05 probability level.

Individuals with a single wild-type version of either FAD2-1A or FAD2-1B in combination with three mutant FAD2-1 alleles (Aabb or aaBb) contained

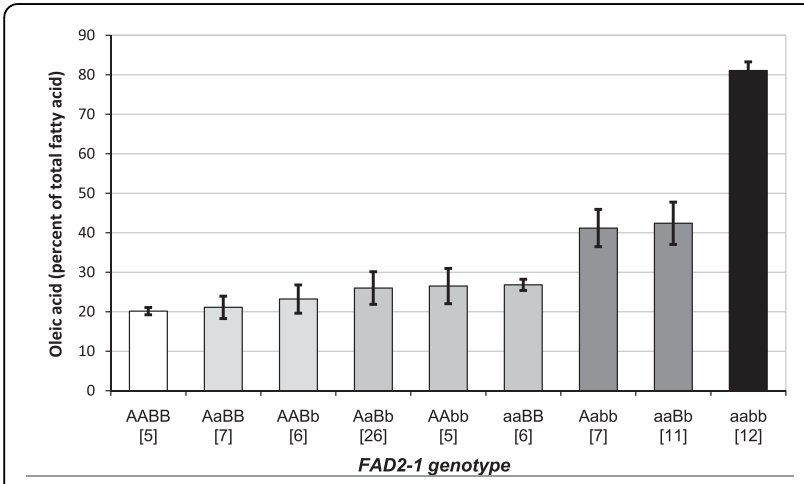

Figure 6 Seed oleic acid phenotype and FAD2-1 genotype association analysis for $F_{2}$ seeds of population 4 . Average oleic acid content of $F_{2}$ soybean seeds produced in Costa Rica from the segregating population developed from the cross $17 \mathrm{D} \times$ S08-14788, population 4. X-axis labels indicate a the soybeans grouped by FAD2-1A and FAD2-1B genotype. $A A=$ wild-type $F A D 2-1 A$ alleles, a $=$ mutant $S 117 \mathrm{~N} F A D 2-1 A$ alleles derived from $17 \mathrm{D}, \mathrm{BB}=$ wild-type FAD2-1B alleles, $\mathrm{bb}=$ mutant P137R FAD2-1B alleles derived from $\mathrm{P}$ 283327; brackets surround the number of soybean seed samples represented in the genotype class. Error bars indicate plus and minus one standard deviation from the mean. Oleic acid phenotype data of each genotype class is the mean of oleic acid content as a percentage of the total fatty acid content of the oil of all lines carrying the FAD2-1 genotype. approximately $40 \%$ oleic acid. No seeds from any of the other FAD2-1 genotypes contained oleic acid levels above $49 \%$ of the seed oil. Individuals with two or more wild-type $F A D 2-1$ alleles contained oleic acid content with a range of $18-47 \%$ of the seed oil.

The necessity of the homozygous FAD2-1A and FAD2$1 B$ mutant combination requirement for the high oleic acid phenotype was confirmed in an independent analysis of FAD2-1 genotype and fatty acid phenotype of field produced $\mathrm{F}_{2}$ seeds that contained homozygous $F A D 2-1 A$ $\triangle$ alleles but which were segregating for $F A D 2-1 B$ P137R alleles (Population 5). While the average oleic acid level of those seeds with the FAD2-1 aabb genotype was $82.5 \%$, FAD2-1 aaBb seeds averaged 55.4\%; FAD2-1 aaBB seeds averaged $43.4 \%$ oleic acid in the seed oil. The presence of a single wild-type version of the $F A D 2-1 B$ allele also prevented a high oleic acid content in the seed oil, although the magnitude of the difference was greater for the $F_{2}$ seeds from Population 4.

\section{The high oleic acid phenotype is stable in plants grown in alternate environments}

Some of the high oleic acid soybean lines developed in this study demonstrated stability for the high oleic acid trait when grown in different environments (Table 1). Of the three environments, Costa Rica typically has the warmest temperatures during seed development, followed by the Portageville, MO environment; the Columbia, MO environment is the coolest of the three environments during seed development $[9,10]$. The differences in the oleic acid contents between environments when the FAD2-1B P137R alleles were present were minor. Soybean lines with genotype FAD2-1aabb of population 2 and 4 produced more than $80 \%$ oleic acid content in Costa Rica and Portageville, MO environments, and the oleic acid level was an average of 2$4 \%$ lower when grown in the Columbia, MO environment. It is notable that the variation in the phenotype was narrow in all of the environments. In contrast, the FAD2-1aabb soybean lines of population 3 containing the $F A D 2-1 B$ I143T alleles had lower and more variable oleic acid content in the cooler environments and failed to produce a high oleic acid phenotype in either the Columbia, MO or Portageville, MO environments.

\section{Full fatty acid profiles and total oil and protein content}

The full fatty acid profiles of the seeds of contrasting FAD2-1 genotypic classes produced from Populations 2, 3 , and 4 in this study revealed additional alterations in palmitic acid, linoleic acid, and linolenic acid content (Table 2). As expected for a major decrease in seed expressed FAD2 enzyme activity that results in an accumulation of oleic acid, the FAD2 reaction products linoleic acid and linolenic acid were dramatically reduced in 
Table 1 Phenotype of different combinations of mutant FAD2-1A and mutant FAD2-1B produced in three environments

\begin{tabular}{|c|c|c|c|c|c|}
\hline \multirow[t]{2}{*}{ Population } & \multirow[b]{2}{*}{ FAD2-1A } & \multirow[b]{2}{*}{ FAD2-1B } & \multicolumn{3}{|c|}{ Oleic acid content (percent of total fatty acid) } \\
\hline & & & Costa Rica ${ }^{1}$ & Portageville, $\mathrm{MO}^{2}$ & Columbia, $\mathrm{MO}^{3}$ \\
\hline 2 & $\Delta$ & P137R & $81.4 \pm 5.7^{F 5}$ & $82.2 \pm 1.2^{F 7}$ & $79.1 \pm 1.3^{\mathrm{F} 8}$ \\
\hline 3 & $\Delta$ & 1143T & $80.0 \pm 4.0^{F 5}$ & $65.0 \pm 4.3^{F 7}$ & $58.7 \pm 7.7^{F 8}$ \\
\hline 4 & $\mathrm{~S} 117 \mathrm{~N}$ & P137R & $81.1 \pm 2.2^{F 2}$ & $81.7 \pm 2.1^{\mathrm{F3}}$ & $77.3 \pm 2.0^{\mathrm{F3}}$ \\
\hline
\end{tabular}

${ }^{1}$ Research station in Costa Rica. Seeds of $F_{5}$ generation of population of 2 and 3 were produced in winter 2006-2007, while $F_{2}$ seeds of population 4 were produced in winter 2008-2009. ${ }^{2}$ Plants were grown in Delta Research Center, seeds of $F_{7}$ generation of the populations 2 and 3 were produced in summer 2008 and $F_{3}$ generation of population 4 was produced in summer 2009. ${ }^{3}$ All of the plants were grown summer 2009 at the Bradford Research \& Extension Center, Columbia MO

the high oleic $F A D 2-1 A$ and $F A D 2-1 B$ homozygous mutant lines when either of the FAD2-1A mutations were present along with the $F A D 2-1 B$ P137R or I143T alleles.

By evaluating the proportions of oleic, linoleic, and linolenic acids present in the oil extracted from mature

Table 2 Fatty acid profiles for different homozygous FAD2-1 genotypes in four segregating populations

\begin{tabular}{|c|c|c|c|c|c|}
\hline \multicolumn{6}{|c|}{ Fatty Acid } \\
\hline & $16: 0$ & $18: 0$ & $18: 1$ & $18: 2$ & $18: 3$ \\
\hline \multicolumn{6}{|c|}{ Population 1 (Jake ${ }^{1} \times$ PI 283327) } \\
\hline $\mathrm{BB}^{2}(\mathrm{n}=24)$ & $12.2 \pm 0.9$ & $3.9 \pm 0.5$ & $20.5 \pm 2.6$ & $53.4 \pm 2.8$ & $10.0 \pm 0.3$ \\
\hline $\mathrm{bb}(\mathrm{n}=30)$ & $11.2 \pm 0.7$ & $3.8 \pm 0.6$ & $29.4 \pm 6.0$ & $47.0 \pm 5.1$ & $8.7 \pm 0.5$ \\
\hline \multicolumn{6}{|c|}{ Population 2 (M23 × PI283327) } \\
\hline $\operatorname{AABB}(n=5)$ & $12.3 \pm 0.5$ & $3.7 \pm 0.4$ & $19.9 \pm 3.3$ & $55.4 \pm 2.7$ & $8.7 \pm 1.0$ \\
\hline AAbb $(n=5)$ & $11.0 \pm 0.5$ & $3.9 \pm 0.4$ & $30.8 \pm 5.2$ & $45.9 \pm 4.6$ & $8.5 \pm 0.9$ \\
\hline $\mathrm{aaBB}(\mathrm{n}=14)$ & $10.8 \pm 0.8$ & $3.8 \pm 0.6$ & $39.4 \pm 5.7$ & $37.1 \pm 4.8$ & $8.9 \pm 1.2$ \\
\hline aabb $(n=16)$ & $7.9 \pm 0.7$ & $3.7 \pm 0.6$ & $82.2 \pm 1.2$ & $2.3 \pm 0.6$ & $3.9 \pm 0.5$ \\
\hline \multicolumn{6}{|c|}{ Population $3(\mathrm{M} 23 \times$ PI 567189A) } \\
\hline $\operatorname{AABB}(n=11)$ & $12.5 \pm 0.9$ & $2.9 \pm 0.4$ & $26.3 \pm 7.4$ & $51.4 \pm 6.4$ & $6.1 \pm 1.2$ \\
\hline AAbb $(n=3)$ & $12.4 \pm 0.8$ & $2.8 \pm 0.4$ & $31.1 \pm 4.5$ & $47.5 \pm 3.3$ & $6.1 \pm 1.0$ \\
\hline $\operatorname{aaBB}(\mathrm{n}=1)$ & $10.3 \pm 0.6$ & $2.8 \pm 0.3$ & $48.2 \pm 7.2$ & $32.5 \pm 6.1$ & $6.2 \pm 0.9$ \\
\hline aabb $(n=16)$ & $8.4 \pm 0.8$ & $2.6 \pm 0.4$ & $80.0 \pm 4.0$ & $5.0 \pm 3.0$ & $3.8 \pm 0.6$ \\
\hline \multicolumn{6}{|c|}{ Population $4 \mathrm{~F}_{2}(17 \mathrm{D} \times \mathrm{S08}-14788)$} \\
\hline $\operatorname{AABB}(n=5)$ & $12.3 \pm 0.9$ & $3.2 \pm 0.3$ & $20.1 \pm 0.9$ & $55.7 \pm 1.0$ & $8.7 \pm 0.6$ \\
\hline AAbb $(n=5)$ & $12.1 \pm 1.0$ & $3.4 \pm 0.5$ & $26.5 \pm 4.5$ & $47.8 \pm 3.7$ & $10.2 \pm 0.9$ \\
\hline aaBB $(n=6)$ & $11.7 \pm 0.3$ & $3.0 \pm 0.2$ & $26.8 \pm 1.4$ & $48.2 \pm 0.7$ & $9.9 \pm 0.5$ \\
\hline aabb (n = 12) & $7.8 \pm 0.5$ & $3.1 \pm 0.2$ & $81.1 \pm 2.2$ & $3.2 \pm 1.4$ & $4.9 \pm 0.6$ \\
\hline \multicolumn{6}{|c|}{ Population $4 \mathrm{~F}_{2: 3}(17 \mathrm{D} \times \mathrm{S08}-14788)$} \\
\hline $\mathrm{AABB}(\mathrm{n}=5)$ & $9.6 \pm 0.6$ & $3.9 \pm 0.4$ & $22.4 \pm 2.9$ & $56.0 \pm 2.8$ & $8.2 \pm 0.9$ \\
\hline AAbb $(n=4)$ & $10.5 \pm 0.5$ & $3.8 \pm 0.3$ & $23.1 \pm 2.5$ & $54.0 \pm 2.6$ & $8.6 \pm 0.5$ \\
\hline $\mathrm{aaBB}(\mathrm{n}=6)$ & $9.3 \pm 0.6$ & $3.2 \pm 0.3$ & $35.0 \pm 7.8$ & $42.9 \pm 5.9$ & $9.6 \pm 2.2$ \\
\hline aabb $(n=11)$ & $6.9 \pm 0.4$ & $3.2 \pm 0.2$ & $77.3 \pm 2.0$ & $6.3 \pm 1.5$ & $6.3 \pm 0.6$ \\
\hline \multicolumn{6}{|c|}{ 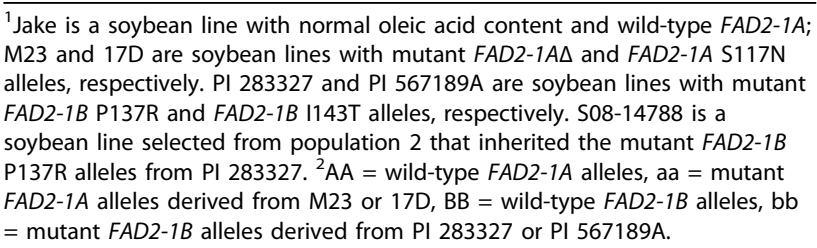 } \\
\hline
\end{tabular}

seeds, the relative in vivo FAD2 and FAD3 desaturase activities of the developing seeds were estimated for the contrasting homozygous FAD2-1 genotypes from each population. The FAD2-1 AABB genotypes contained estimated FAD2 desaturase activities (the sum of the final linoleic and linolenic acid contents divided by the sum of final oleic, linoleic, and linolenic acid contents, expressed as a percent) of 76\%, 76\%, and 74\% for Population 2, Population 3, and Population 4, respectively. The FAD2-1 aabb genotypes contained FAD2 desaturase activities of $7 \%, 10 \%$, and $14 \%$, for Population 2, Population 3, and Population 4, respectively. Also noted is that the accumulation of linolenic acid follows a different pattern for the FAD2-1 aabb mutant lines compared to the FAD2-1 AABB lines, with increased estimated FAD3 desaturase activity (final linolenic acid content divided by the sum of final linoleic and linolenic acid contents, expressed as a percent) for the FAD2-1 mutant lines.

While no significant differences were observed for the stearic acid levels in the contrasting FAD2-1 genotypes, the $F A D 2-1$ aabb mutant lines consistently produced lower palmitic acid levels than lines with the FAD2-1 AABB genotype. The most dramatic change was for Population 2. In that case, the content of palmitic acid was $7.9 \%$ for the $F A D 2-1$ aabb mutant lines compared to $12.3 \%$ for the FAD2-1 AABB lines.

Because of the concern that improvement in fatty acid profiles might have negative impacts on the total oil and protein profiles of the seeds, we also evaluated the protein and oil contents for the field produced $\mathrm{F}_{2: 3}$ seeds from Population 4. There were no significant differences in the protein or oil contents among the different homozygous FAD2-1 genotypes, or with those lines compared to either Williams 82 or the 17D parental line. The $F A D 2-1 B$ P137R allele donor parental line had a minor decrease in the average oil content and the highest mean protein content of all of the lines examined.

\section{Discussion and Conclusions}

Increasing the oleic acid content in soybean seed oil is one of the most effective and efficient ways to enhance 
the nutritional value and practical utilization of soybean oil. However, the previously characterized sources of elevated oleic acid soybean involved mutation of the FAD2-1A gene alone, which failed to achieve oleic acid levels stable above $60 \%$ of the oil $[19,21,32]$ or utilized approaches that have proven to have limited usage in soybean breeding due to the complexity of the trait [23]. A transgenic approach was successful in downregulation of the FAD2-1 genes leading to high oleic acid levels in the oil $[25,26]$. We have demonstrated here that an allele of the $F A D 2-1 B$ gene containing a conserved amino acid substitution is responsible for the elevated oleic acid content in PI 283327, since soybean lines inheriting the homozygous mutant alleles have higher levels of oleic acid compared to lines inheriting wild type $F A D 2-1 B$ alleles. The PI 567189A FAD2-1B allele, which contains a very rare amino acid substitution, was also predicted to have a negative impact on the enzyme activity and function.

The most significant finding of this research was that the mutant $F A D 2-1 B$ alleles from either PI 283327 or PI 567189A not only contribute to the elevated oleic acid content in soybean seed oil of the two germplasm accessions but also enable the accumulation of oleic acid content nearly four-fold, to more than $80 \%$ of the oil, in soybean seeds when combined with different sources of a mutant FAD2-1A gene. Our results define the requirement of the two major contributors to the FAD2 enzyme activity present in developing soybean seeds, FAD2-1A and FAD2$1 B$. Other $F A D 2$ genes exist in the soybean genome and some are expressed during seed development. However, when FAD2-1A and FAD2-1B are non-functional, very little FAD2 activity appeared to remain in developing seeds, as evidenced by the minor accumulation of linoleic and linolenic acid in the seed oil of the lines containing the $F A D 2-1 A$ and $F A D 2-1 B$ mutant combinations.

Our study demonstrated that different types of FAD2$1 A$ mutant alleles bring about a similar high level of oleic acid content in the oil of soybean seeds when combined with the P137R FAD2-1B allele from PI 283327. M23 has lost its $F A D 2-1 A$ alleles during the X-ray treatment while $17 \mathrm{D}$ carries the alleles with a mutagenesisinduced mutation that appeared to be less effective in accumulating oleic acid in the seed oil $[19,21]$. Hence, it was assumed that 17D would be less useful than M23 in creating a high oleic acid trait. In contrast to what we expected, the $F A D 2-1 A$ alleles from 17D were able to combine with mutant $F A D 2-1 B$ to produce oleic acid levels similar to those when M23 was the source of the deleted FAD2-1A gene, and the phenotype was stable across the different environments evaluated. We conclude that for the FAD2-1A gene from 17D the encoded enzyme was unable to function appropriately, so when in combination with the mutant P137R FAD2-1B allele, the conversion of oleic acid precursors to linoleic acid precursors was almost completely blocked. Presumably there is some compensatory difference in the activity of the functional FAD2-1B when the S117N allele of FAD2-1A was present compared to the situation from M23 when $F A D 2-1 A$ is deleted.

The occurrence of soybean lines with the high oleic acid phenotype in population 4 fit a model of two independent recessive genes segregating with very little evidence for additional modifier genes, demonstrating that in this circumstance only FAD2-1A and FAD2-1B are contributing to the high oleic acid content in soybean seed oil. Based on our results, we hypothesize that any soybean line carrying a null or severely mutated FAD2$1 A$ allele that is crossed with PI 283327 is likely to produce individual soybean seeds in the $\mathrm{F}_{2}$ progeny with at least $80 \%$ oleic acid content of the seed oil.

Although there is evidence of influence of temperature on the soybean seed oleic acid content $[9,10]$, two of our three high oleic acid soybean genotypes proved to be capable of producing a high and stable oleic acid content in three environments. Moreover, there was no reduction in oil and protein content in the evaluated high oleic acid soybean lines. Soybean lines with the combination of FAD2-1A $\triangle$ and FAD2-1B I143T alleles from population 3 failed to produce the high oleic acid phenotype when grown in the non-tropical environments. A possible explanation is the mutation in the $F A D 2-1 B$ allele of PI 567189 A encodes at least nominal enzyme function. This explanation is supported by the fact that the I143T substitution is in a less conserved amino acid of the FAD2 enzyme than the P137R substitution. Other than that, our high oleic acid soybean lines showed a reduction of $4 \%$ at most when they were grown in the cooler environment, with a small variation in the oleic acid content. It will be necessary to test the performance of these high oleic acid soybean lines in the main North American soybean growing locations in more northern latitudes. The mutant FAD2-1A and $F A D 2-1 B$ alleles will have to be combined in soybean lines with the appropriate maturity for those experiments to be conducted. However, based on the stability of the trait that we have observed so far, any reduction of oleic acid content due to the environment is likely to be minor because very little FAD2 enzyme activity remains in developing seeds in the mutant FAD2-1A and $F A D 2-1 B$ lines. An additional factor is that the end use market has not matured sufficiently to define the exact oleic acid content desired for different oil uses. Another question that should be addressed is whether the trait will affect yield or other agronomic traits. It has been reported that the transgenic soybean lines with the FAD2-1 genes being silenced did not show any yield drag or abnormal physiology characteristics [26]. 
The relative contribution of FAD2-1A and FAD2-1B in oleic acid accumulation in soybean oil could not be fully explored in this study due to the lack of a true null allele of $F A D 2-1 B$. Previous research has indicated that $F A D 2-1 B$ is expressed at a higher level than $F A D 2-1 A$, and that FAD2-1B is more stable than FAD2-1A when expressed recombinantly in yeast $[5,7,19,20]$. Our sequencing results of $F A D 2-1 A$ and $F A D 2-1 B$ alleles from 24 PIs with elevated oleic acid content revealed that the FAD2-1A gene sequence is much more conserved than $F A D 2-1 B$ (data not shown). If the assumption is made that the P137R allele of FAD2-1B is nonfunctional, then the contribution of FAD2-1A to the FAD2 desaturase activity in developing seeds appears greater than that of FAD2-1B, although the variability in the fatty acid profiles for the lines that contain functional versions of FAD2-1A or FAD2-1B obscures the contribution from each allele. If the P137R allele of $F A D 2-1 B$ retains some activity, then it could account for only one tenth of the original FAD2 activity present (in population 2, for the aabb genotypes containing a null FAD2-1A compared to the AABB genotypes).

Traditional breeding has been used previously to produce soybean with up to $70 \%$ oleic acid content in the seed oil [33]. However, the phenotype is not consistent across environments, and the genetics of the trait is not very well understood, which limits the usage of these soybean lines. Practices to boost oleic acid content in soybean to more than $80 \%$ have been achieved by means of suppression of the expression level of FAD2 genes, and as a result, transgenic high oleic soybeans were produced [25]. Commercial release of transgenic plants still has to overcome regulatory hurdles, and production and importation of transgenic plants remain unacceptable in various countries.

Our research results have demonstrated the capacity to develop soybeans containing more than $80 \%$ oleic acid in the oil based on very simple genetic manipulation, the combination of two recessive genes. As part of this research, we developed molecular marker assays that allow the selection of the desired mutant FAD2-1A and $F A D 2-1 B$ alleles, even when they are present in the heterozygous state. Molecular marker selection thus eliminates the time necessary to produce an extra generation of plants that must be screened for the fatty acid phenotype. Simple genetics combined with perfect molecular marker assays will make it possible for soybean breeders to quickly incorporate the high oleic acid trait in their breeding programs. The resulting high oleic acid soybean lines can then be efficiently developed and released as cultivars to producers. Also, because only two genes control the vast majority of the high oleic acid phenotype, other genes may be added to enhance soybean oil quality such as low linolenic acid, low allergens, or a growing list of traits involved in soybean meal quality [34]. In the short term, examination of the stability of the high oleic acid soybean lines across different environments is of particular interest. Also, the soybean lines with the high oleic acid trait should be used for development of soybean varieties with favorable agronomic traits including high yield.

In conclusion, this research demonstrates that when mutant alleles of FAD2-1A and FAD2-1B are combined together by means of traditional plant breeding, they can significantly enhance the oleic acid content of the oil, up to $80 \%$, providing a means for the development of soybean varieties with superior oil quality.

\section{Methods}

\section{Population development}

Recombinant inbred lines (RIL) from population $1\left(\mathrm{~F}_{6}\right.$ RIL of Jake $\times$ PI 283327), $2\left(\mathrm{~F}_{2: 6}\right.$ and $\mathrm{F}_{2: 7}$ RIL of M23 $\times$ PI283327) and $3\left(\mathrm{~F}_{2: 5}\right.$ and $\mathrm{F}_{2: 7}$ RIL of M23 $\times$ PI 567189 A) were created at the same time. Three crosses were made in summer 2005 at the Delta Research Center at Portageville, MO including Jake $\times$ PI 283327, M23 $\times$ PI 283327 and M23 × PI 567189A. PI 283327 and PI 567189A are two elevated oleic acid lines with maturity group V and IV, respectively (GRIN USDA), while Jake is a conventional high yielding soybean in group $\mathrm{V}$ that contains a typical oleic acid content [31]. M23 was selected for elevated oleic acid after mutagenesis of the cultivar Bay [35]. In 2005 and early 2006, $F_{1}$ seeds were advanced to the $\mathrm{F}_{2}$ generation in Costa Rica. Each RIL tracing to a single $F_{2}$ plant except population 1 was also advanced in Costa Rica from 2006 to 2007 for $F_{5}$ seeds. In 2007, a bulk of five seeds from each RIL in each population was analyzed to obtain fatty acid profile for the Costa Rica location. Population 1 was grown in Portageville, $\mathrm{MO}$ to produce $\mathrm{F}_{7}$ seeds. Population 2 was grown in Portageville, $\mathrm{MO}$ to produce $\mathrm{F}_{6}$ seeds, and then soybean RILs with more than $60 \%$ oleic acid were advanced to the $F_{7}$ generation. In population 3 , only $F_{5}$ RILs producing more than $60 \%$ oleic acid were selected to generate $F_{7}$ seeds at Portageville, $\mathrm{MO}$ in subsequent generations.

Population 4 was developed from the cross $17 \mathrm{D} \times$ S08-14788 which was created in the summer of 2008 at Portageville, MO. S08-14788 was selected from population 1 because it carried the FAD2-1B P137R mutant alleles derived from PI 283327. 17D is an elevated oleic soybean line developed by mutagenesis with $35 \%$ oleic acid content [21]. True $F_{1}$ seeds were sent to Costa Rica and $F_{2}$ seeds were produced in the winter 2009. $F_{2: 3}$ seeds were produced in Columbia, MO and Portageville, MO during the summer 2009 growing season.

Population 5 was initiated in summer 2008 at Portageville, MO. Soybean line KB07-1\#123 was crossed with 
soybean line \#93 from population 2. Soybean line \#93 (> $80 \%$ oleic acid) was genotyped to contain the FAD2-1A $\triangle$ alleles from M23 and the $F A D 2-1 B$ P137R alleles derived from PI 283327. KB07-1\#123 is a soybean line with the pedigree [W82 $\times(\mathrm{M} 23 \times 10-73)]$. This soybean line was selected to contain three mutant alleles affecting the fatty acid profile, including $F A D 2-1 A \triangle$ alleles from M23, and mutant $F A D 3 A$ and $F A D 3 C$ alleles from soybean line $10-73$ [21,36]. $F_{1}$ seeds were genotyped to confirm the heterozygosity and then advanced to obtain $\mathrm{F}_{2}$ seeds in summer 2009 at Bradford Research and Extension Center, Columbia MO.

In 2008, populations 1 and 2 were grown in Portageville, MO to produce the seeds analyzed for fatty acids in figures 2 and 3. In 2009, population 4 was grown in Columbia, MO to produce the seeds analyzed for fatty acid analysis in figure 5 . Data in figure 4 was from $F_{5}$ seeds of population 3 produced in Costa Rica. In addition, five lines with the highest oleic acid content from populations 2 and 3 were grown in Columbia, MO in 2009. Similarly, four to eleven lines from each of four combinations of homozygous FAD2-1A and FAD2-1B genes from population 4 were grown in Columbia $\mathrm{MO}$ and selected lines from population 4 were grown in Portageville, MO in 2009.

\section{DNA isolation and PCR for sequencing of FAD2-1A and FAD2-1B}

Genomic DNA was isolated from approximately $30 \mathrm{mg}$ of dry seeds using the DNeasy Plant Mini Kit (Qiagen, Inc., Valencia, CA) and used at 5 to $50 \mathrm{ng}$ per PCR reaction. PCR was carried out using Ex Taq according to manufacturer's recommendation (Takara, Otsu, Shiga, Japan) in a PTC-200 thermocycler (MJ Research/BioRad, Hercules, CA). Primers for FAD2-1A: forward was ACTGCATCGAATAATACAAGCC and reverse was TGATATTGTCCCGTGCAGC. Primers for FAD2-1B: forward was CCCGCTGTCCCTTTTAAACT and reverse was TTACATTATAGCCATGGATCGCTAC. PCR was programmed as the following: $95^{\circ} \mathrm{C}$ for $5 \mathrm{~min}$ utes followed by 35 cycles of $95^{\circ} \mathrm{C}$ for 30 seconds, $60^{\circ} \mathrm{C}$ for 30 seconds, and $72^{\circ} \mathrm{C}$ for 1 minute 30 seconds. PCR products were examined for size by running on Flashgel (Lonza Group Ltd., Switzerland) for 5 minutes. PCR products were then isolated with the Qiaprep Spin Miniprep kit (Qiagen, Inc.) and sequenced at the University of Missouri DNA Core facility.

\section{Sequence analysis}

Sequences were aligned using Multiple Sequence Alignment by CLUSTALW http://align.genome.jp/, and evaluated for variant nucleotides between 'Williams 82' reference http://www.phytozome.net/soybean and the PIs. Protein translation was conducted using ExPaSy http://ca. expasy.org/tools/dna.html and protein alignment was done using Multiple Sequence Alignment program.

\section{FAD2-1B allele specific molecular marker assay}

SimpleProbe assays were based on the disassociation kinetics of SimpleProbe obligonucleotides (Roche Applied Sciences) to be exactly complimentary to the 'Williams 82' reference sequence. SimpleProbe was purchased from Roche Applied Sciences. The FAD2-1B probe consists of 5 -Fuorescence-AGTCCCTTATTTCTCATGGAAAATAAGC-Phosphate-3'. C > G mutation (P137R allele from PI 283327) is indicated by underline and $\mathrm{T}>\mathrm{C}$ mutation (I143T allele from PI 567189A) is indicated by bold font. Primers used to generate template for Simpleprobe genotyping assay were designed by aligning the $F A D 2-1 A$ and $F A D 2-1 B$ region containing the SNPs. Primers were selected to be as close as possible to the SNPs while differing in at least 3 nucleotides between the two genes to specifically amplify the targeted region in FAD2-1B. Genotype reactions used asymmetric PCR to generate additional single stranded DNA to which the Simpleprobe could bind with less competition from the opposite amplification stand. Because the FAD2-1B SNPs found in the two PIs were only a few nucleotides apart, the SimpleProbe was designed to detect both of the SNPs. Genotyping reactions were performed with a 5:2 asymmetric mix of primers (5'-ACTGCATCGAATAATACAAGCC-3' at $2 \mu \mathrm{M}$ final concentration, and 5'-TGATATTGTCCCGTCCAGC-3' at $5 \mu \mathrm{M}$ final concentration). Reactions were carried out in $20 \mu \mathrm{l}$; containing template, primers, 0.2 $\mu \mathrm{M}$ final concentration of SimpleProbe, buffer $(40 \mathrm{mM}$ Tricine-KOH $[\mathrm{pH} 8.0] 16 \mathrm{mM} \mathrm{MgCl}_{2}, 3.75 \mu \mathrm{g} \mathrm{ml}^{-1}$ BSA,), 5\% DMSO, $200 \mu \mathrm{M}$ dNTPs, and 0.2X Titanium Taq polymerase (BD Biosciences, Palo Alto, CA). Genotyping reactions were performed using a Lightcycler 480 II real time PCR instrument (Roche), using the following PCR parameters: $95^{\circ} \mathrm{C}$ for 5 minutes followed by 40 cycles of $95^{\circ} \mathrm{C}$ for 20 seconds, $60^{\circ} \mathrm{C}$ for 20 seconds, $72^{\circ} \mathrm{C}$ for 20 seconds, and then a melting curve from $50^{\circ} \mathrm{C}$ to $70^{\circ} \mathrm{C}$. When DNA from PI 283327 and PI 567189A is amplified with gene specific primers and used in melting curve analysis with the SimpleProbe, a mismatch between the Simpleprobe and the amplicon results in altered disassociation kinetics. Each genotype produced a characteristic melting profile, as measured by Tm of the negative first derivative of the disappearance of fluorescent signal. PI 283327 and all soybean lines with an identical $F A D 2-1 B$ allele genotype have a characteristic peak at $56.7^{\circ} \mathrm{C}$, while the PI 567189A FAD2- $1 B$ allele genotype yielded a characteristic peak at $60.2^{\circ} \mathrm{C}$. M23 and Jake (wild-type $F A D 2-1 B$ ) have a peak at $62.5^{\circ} \mathrm{C}$. Heterozygous individuals's genotype showed two peaks at either $56.7^{\circ} \mathrm{C}$ or $60.2^{\circ} \mathrm{C}$ and $62.5^{\circ} \mathrm{C}$. 
FAD2-1A allele specific molecular marker assay for 17D The assay was conducted as described by Dierking 2009 [21].

\section{FAD2-1A allele specific molecular marker assay for M23}

An allele specific molecular marker assay was developed to distinguish soybean lines with deletion of FAD2-1A $(F A D 2-1 A \triangle$ alleles from M23) and the soybean lines with the presence of one (heterozygous) or two FAD2$1 A$ alleles.

The reactions contain two primer pairs: one pair specific for $F A D 2-1 A$ gene amplification and one pair specific for phosphoenolpyruvate carboxylase 16 (PEPC16) gene amplification, as an internal amplification control. The FAD2-1A primer pair has forward primer T2AF: (5'-ATCTTTAGATTTTTCACTACCTGGTTTAAA ATTGAGGGATTG-3') and reverse primer HOLL1 (5'CTTTGCTAGACCCTGTGTCAAAGTATAAAC-3'). The PEPC16 primer pair has forward primer PEPC16fwd (5'-TTCCTTTATCAGAAATAACGAGTTTAGCT-3') and reverse primer PEPC16rev (5TGTCTCATTTTGCGGCAGC-3').

Reactions were carried out in $15 \mu \mathrm{l}$; each primer was at $1.3 \mu \mathrm{M}$ final concentration in reactions containing template, buffer ( $40 \mathrm{mM}$ Tricine- $\mathrm{KOH}$ (pH 8.0), $16 \mathrm{mM} \mathrm{KCl,}$ $3.5 \mathrm{mM} \mathrm{MgCl}_{2}, 3.75 \mu \mathrm{g} \mathrm{ml}^{-1}$ BSA, $200 \mu \mathrm{M}$ dNTPs), 5\% DMSO, $1.25 \mu \mathrm{M}$ EvaGreen (Biotium Inc., Hayward, CA) and 0.2X Titanium Taq polymerase (BD Biosciences, Palo Alto, CA). PCR parameters on a DNA Engine Opticon 2 (MJ Research/Bio-Rad) were as follows: $95 \mu \mathrm{C}$ for 5 minutes followed by 35 cycles of $95 \mu \mathrm{C}$ for 20 seconds, $64 \mu \mathrm{C}$ for 20 seconds, $72 \mu \mathrm{C}$ for 20 seconds, and then a melting curve from $70 \mu \mathrm{C}$ to $85 \mu \mathrm{C}$. The fluorescence was read after each cycle and every $0.2 \mu \mathrm{C}$ with a one second hold during the melt with excitation at 470-505 $\mathrm{nm}$ and detection at 523-543 $\mathrm{nm}$. Each genotype produced a product with a characteristic melting profile, as measured by $\mathrm{Tm}$ of the negative first derivative of the disappearance of fluorescent signal. Homozygous wildtype $F A D 2-1 A$ alleles and heterozygous samples produced a peak at $76^{\circ} \mathrm{C}$ and possibly another peak at $78^{\circ} \mathrm{C}$; homozygous mutant alleles (FAD2-1A $\triangle$ ) only produced a peak at $78 \mu \mathrm{C}$. Templates for PCR were either genomic DNA samples isolated using the DNeasy Plant Mini Kit (Qiagen, Inc., Valencia, CA) or $1.2 \mathrm{~mm}$ washed FTA (Whatman) card punches prepared from leaves according to the manufacturer's instructions.

\section{Fatty acid, protein and oil determination}

The method of gas chromatography of total fatty acid methyl esters of extracted oil was used to examine the fatty acid profiles of all samples [37]. The individual fatty acid contents are reported as the relative percents of palmitic, stearic, oleic, linoleic, and linolenic acids in the extracted oil. For the RIL populations, individual whole crushed seeds for each soybean line were used as samples for fatty acid determination, except the FAD2laabb lines from population 3 produced in Costa Rica, for which a bulk sample of 5 seeds was analyzed for each line. For the $F_{2}$ seeds from population 4 and population 5 , seed chips were used by removing a "chipped" portion of the seed opposite the embryo with a scalpel for fatty acid analysis such that the remainder of the seed containing the embryo could be germinated.

Protein and oil contents were determined for seeds of $F_{2: 3}$ lines for population 4 using NIR spectroscopy [38].

\section{Population genotyping}

For every RIL population, a seed from each line was germinated in a germination package. Eight to ten days later, a small developing trifoliate leaf was excised and pressed onto an FTA card for DNA storage. Based on fatty acid profiles obtained from the other five seeds, to obtain a set of the contrasting homozygous FAD2-1A and $F A D 2-1 B$ genotypes, selected lines of the three populations Jake $\times$ PI 283327, M23 $\times$ PI 283327, M23 $\times$ PI 567189A, were genotyped with all allele-specific assays as described. For the $\mathrm{F}_{2}$ seeds of population 4 , 200 seeds were chipped, the small portion of each without hypocotyls was sent for fatty acid profiling, the remaining chipped seed was germinated to collect DNA for genotyping. Not all samples were genotyped.

\section{Acknowledgements}

The authors wish to acknowledge excellent technical assistance provided by Paul Little and Christine Cole. Ed Cahoon provided the results for the recombinant functional expression of variant alleles of FAD2-1B. Funding for this research was provided by the University of Missouri, the United Soybean Board, the Missouri Soybean Merchandising Council, and USDA/ARS.

\section{Author details}

'University of Missouri, Division of Plant Sciences, 110 Waters Hall, Columbia, MO 65211 USA. ${ }^{2}$ Division of Plant Biosciences, Kyungpook National University, Daegu 702-701, Republic of Korea. ${ }^{3}$ University of Missouri, Division of Plant Sciences, University of Missouri-Delta Research Center, Portageville, MO 63873 USA. ${ }^{4}$ USDA-ARS, Plant Genetics Research Unit, 110 Waters Hall, Columbia, MO 65211 USA.

\section{Authors' contributions}

TAP and JDL contributed equally to this research. JDL and JGS selected the germplasm lines for analysis and developed the populations. TAP and KDB designed and conducted the molecular biology analyses and the association analyses. TAP and KDB drafted the manuscript with contributions and edits from JDL and JGS. All authors read and approved the final manuscript.

Authors' information

Dr Bilyeu can also be contacted at bilyeuk@missouri.edu.

Received: 3 May 2010 Accepted: 9 September 2010

Published: 9 September 2010

\section{References}

1. Ascherio A, Willett WC: Health effects of trans fatty acids. American Journal of Clinical Nutrition 1997, 66(Suppl 4):1006-1010. 
2. Raneses AR, Glaser LK, Price JM, Duffield JA: Potential biodiesel markets and their economic effects on the agricultural sector of the United States. Industrial Crops and Products 1999, 9(2):151-162.

3. Butzen S, Schnebly S: High oleic soybean. Crop Insights 2007, 17(7):3.

4. Okuley J, Lightner J, Feldmann K, Yadav N, Lark E, Browse J: Arabidopsis FAD2 gene encodes the enzyme that is essential for polyunsaturated lipid synthesis. Plant Cell 1994, 6(1):147-158.

5. Schlueter JA, Vasylenko-Sanders IF, Deshpande S, Yi J, Siegfried M, Roe BA, Schlueter SD, Scheffler BE, Shoemaker RC: The FAD2 gene family of soybean:Insights into the structural and functional divergence of a paleopolyploid genome. Crop Science 2007, 47(Supplement_1), S-14-26.

6. Beló A, Zheng P, Luck S, Shen B, Meyer DJ, Li B, Tingey S, Rafalski A: Whole genome scan detects an allelic variant of fad 2 associated with increased oleic acid levels in maize. Molecular Genetics and Genomics 2008, 279:1-10.

7. Tang GQ, Novitzky WP, Griffin HC, Huber SC, Dewey RE: Oleate desaturase enzymes of soybean: evidence of regulation through differential stability and phosphorylation. Plant Journal 2005, 44:433-446.

8. Heppard EP, Kinney AJ, Stecca KL, Miao GH: Developmental and growth temperature regulation of two different microsomal [omega]-6 desaturase genes in soybeans. Plant Physiol 1996, 110(1):311-319.

9. Lee JD, Woolard M, Sleper DA, Smith JR, Pantalone VR, Nyinyi CN, Cardinal A, Shannon JG: Environmental effects on oleic acid in soybean seed oil of Plant Introductions with elevated oleic concentration. Crop Science 2009, 49(5):1762-1768

10. Oliva ML, Shannon JG, Sleper DA, Ellersieck MR, Cardinal AJ, Paris RL, Lee JD: Stability of fatty acid profile in soybean genotypes with modified seed oil composition. Crop Science 2006, 46(5):2069-2075.

11. Knowles PF, Hill AB: Inheritance of fatty acid content in the seed oil of a safflower introduction from Iran. Crop Science 1964, 4:406-409.

12. Liu Q, Singh S, Green A: High-oleic and high-stearic cottonseed oils: nutritionally improved cooking oils developed using gene silencing. Journal of the American College of Nutrition 2002, 21:205S-211S.

13. Bruner $A C$, Jung $S$, Abbott $A G$, Powell $G L$ : The naturally occurring high oleate oil character in some peanut varieties results from reduced oleoyl-PC desaturase activity from mutation of Aspartate 150 to Asparagine. Crop Science 2001, 41(2):522-526.

14. Jung S, Powell G, Moore K, Abbott A: The high oleate trait in the cultivated peanut [Arachis hypogaea L.]. II. Molecular basis and genetics of the trait. Molecular and General Genetics 2000, 263(5):806-811.

15. Patel M, Jung S, Moore K, Powell G, Ainsworth C, Abbott A: High-oleate peanut mutants result from a MITE insertion into the FAD2 gene. Theoretical Applied Genetics 2004, 108:1492-1502.

16. Stoutjesdijk PA, Hurlestone C, Singh SP, Green AG: High-oleic acid Australian Brassica napus and B. juncea varieties produced by cosuppression of endogenous Delta12 desaturases. Biochemical Society Transaction 2000, 28(6):938-940.

17. Hu X, Sullivan-Gilbert M, Gupta M, Thompson SA: Mapping of the loci controlling oleic and linolenic acid contents and development of fad2 and fad3 allele-specific markers in canola (Brassica napus L.). Theoretical Applied Genetics 2006, 113:497-507.

18. Alt J, Fehr W, Welke G, Sandhu D: Phenotypic and molecular analysis of oleate content in the mutant soybean line M23. Crop Science 2005, 45(5):1997-2000

19. Anai T, Yamada T, Hideshima R, Kinoshita T, Rahman SM, Takagi Y: Two high-oleic-acid soybean mutants, M23 and KK21, have disrupted microsomal omega- 6 fatty acid desaturase, encoded by GmFAD2-1a. Breeding Sciences 2008, 58:447-452.

20. Sandhu D, Alt J, Scherder C, Fehr W, Bhattacharyya M: Enhanced oleic acid content in the soybean mutant M23 is associated with the deletion in the Fad2-1a gene encoding a fatty acid desaturase. Journal of the American Oil Chemists' Society 2007, 84(3):229-235.

21. Dierking E, Bilyeu K: New sources of soybean seed meal and oil composition traits identified through TILLING. BMC Plant Biology 2009, 9(1):89.

22. Burton JW, Wilson RF, Rebetzke GJ, Pantalone VR: Registration of N984445A mid-oleic soybean germplasm line. Crop Science 2006, 46(2):1010-1012

23. Bachlava E, Dewey RE, Burton JW, Cardinal AJ: Mapping and comparison of quantitative trait loci for oleic acid seed content in two segregating soybean populations. Crop Science 2009, 49:433-442.
24. Monteros MJ, Burton JW, Boerma HR: Molecular mapping and confirmation of QTLs associated with oleic acid content in N00-3350 soybean. Crop Science 2008, 48(6):2223-2234.

25. Buhr T, Sato S, Ebrahim F, Xing A, Zhou Y, Mathiesen M, Schweiger B, Kinney A, Staswick P, Clemente T: Ribozyme termination of RNA transcripts down-regulate seed fatty acid genes in transgenic soybean. Plant Journal 2002, 30:155-163.

26. Graef G, LaVallee B, Tenopir P, Tat M, Schweiger B, Kinney A, Gerpen J, Clemente T: A high-oleic-acid and low-palmitic-acid soybean: agronomic performance and evaluation as a feedstock for biodiesel. Plant Biotechnology Journal 2009, 7(5):411-421.

27. Lee J-D, Bilyeu KD, Shannon JG: Genetics and breeding for modified fatty acid profile in soybean seed oil. Journal of Crop Science and Biotechnology 2007, 10:201-210.

28. Bernard R, Cremeens C: Registration of 'Williams 82' Soybean. Crop Science 1988, 28(6):1027-1028.

29. Ramensky V, Bork P, Sunyaev S: Human non-synonymous SNPs: server and survey. Nucleic Acids Research 2002, 30(17):3894-3900.

30. Crooks G, Hon G, Chandonia J-M, Brenner S: WebLogo: A sequence logo generator. Genome Research 2004, 14(6):1188-1190.

31. Shannon JG, Wrather JA, Sleper DA, Robinson RT, Nguyen HT, Anand SC: Registration of 'Jake' Soybean. Journal of Plant Registration 2007, 1:29-30

32. Scherder CW, Fehr WR: Agronomic and seed characteristics of soybean lines with increased oleate content. Crop Science 2008, 48(5):1755-1758.

33. Alt JL, Fehr WR, Welke GA, Shannon JG: Transgressive segregation for oleate content in three soybean populations. Crop Science 2005, 45(5):2005-2007.

34. Bilyeu K, Palavalli L, Sleper DA, Beuselinck P: Molecular genetic resources for development of $1 \%$ linolenic acid soybeans. Crop Science 2006, 46(5):1913-1918

35. Takagi Y, Rahman SM: Inheritance of high oleic acid content in the seed oil of soybean mutant M23. Theoretical Applied Genetics 1996, 92:179-182.

36. Bilyeu K, Palavalli L, Sleper D, Beuselinck P: Mutations in soybean microsomal omega-3 fatty acid desaturase genes reduce linolenic acid concentration in soybean seeds. Crop Science 2005, 45(5):1830-1836.

37. Beuselinck PR, Sleper DA, Bilyeu KD: An assessment of phenotype selection for linolenic acid using genetic markers. Crop Science 2006, 46:747-750.

38. Hartwig RA, Hurburgh CR: Near-infrared reflectance measurement of moisture, protein and oil content of ground crambe seed. Journal of the American Oil Chemists' Society 1990, 67:435-437.

doi:10.1186/1471-2229-10-195

Cite this article as: Pham et al: Mutant alleles of FAD2-1A and FAD2-1B combine to produce soybeans with the high oleic acid seed oil trait. BMC Plant Biology 2010 10:195.

\section{Submit your next manuscript to BioMed Central and take full advantage of:}

- Convenient online submission

- Thorough peer review

- No space constraints or color figure charges

- Immediate publication on acceptance

- Inclusion in PubMed, CAS, Scopus and Google Scholar

- Research which is freely available for redistribution 